\title{
Clinical Significance of the Metastatic Lymph-Node Ratio in Rectal Cancer
}

\author{
Hyoung Chul Park \\ Department of Surgery, Hallym University Sacred Heart Hospital, Hallym University College of Medicine, Anyang, Korea
}

See Article on Page 100-105

This study investigated the prognostic significance of the metastatic lymph-node ratio (LNR) in patients with rectal cancer. Since Berger et al. [1] reported that the ratio of metastatic to examined lymph nodes in stage III colon cancer was associated with survival, the LNR has been considered to provide a prediction of the prognosis. Patients with stage IIIB and IIIC colorectal cancer with 13 or more negative nodes were also demonstrated to show a better prognosis [2]. In addition, as an inadequate number of harvested lymph nodes can result in stage migration, the LNR has come to be recognized as a potentially more accurate prognostic factor. Furthermore, node positive cancer with a high LNR may be related to aggressive features of tumor biology.

Although some studies including Korean patients have reported the prognostic significance of the LNR in patient with isolated rectal cancer [3-6], the results were not well described. For the clinical significance of LNR, a sufficient number of harvested or examined lymph nodes should be considered. Especially, many factors, including preoperative chemoradiation, may affect on that number in patients with rectal cancer. Although this study excluded patients who underwent neoadjuvant treatment, three studies assessed the prognostic value of the LNR in patient with rectal cancer who underwent preoperative treatment [7-9].

Various cutoff points of the LNR for statistically different survivals have been reported in many articles. However, in this study, the authors used initially 0.23 as the cutoff value, but no significant difference. Consequently, authors suggested 0.15 as a new

Correspondence to: Hyoung Chul Park, M.D.

Department of Surgery, Hallym University Sacred Heart Hospital, Hallym University College of Medicine, 22 Gwanpyeong-ro 170beon-gil, Dongan-gu, Anyang 431-796, Korea

Tel: +82-31-380-3041, Fax: +82-31-385-0153

E-mail: greatpa1@hallym.or.kr

(c) 2013 The Korean Society of Coloproctology

This is an open-access article distributed under the terms of the Creative Commons Attribution NonCommercial License (http://creativecommons.org/licenses/by-nc/3.0) which permits unrestricted noncommercial use, distribution, and reproduction in any medium, provided the original work is properly cited. cutoff point to obtain significance [10]. However, this approach has a limitation, the lack of a consensus on an appropriate cutoff value, which could results in a manipulation of the cutoff value without validation.

The LNR was proposed to be superior to the absolute number of metastatic lymph node for prognostic differentiation in stage III colorectal cancer [11-13]. However, many studies on the LNR were designed retrospectively and involved a relatively small number of patients. If the LNR is to have excellent prognostic value, a systematical evaluation involving a large-scale study is needed to establish a more reliable cutoff value of the LNR. In addition, in patients with colorectal cancer, the cutoff point for the LNR could vary with the tumor location and/or the treatment option. For example, the cutoff values for the LNR could be different between right colon cancer and rectal cancer or between rectal cancer with neoadjuvant therapy and without therapy. Thus, establishing an appropriate cutoff LNR value for a specific disease state may be reasonable.

In conclusion, the LNR may a good candidate for an independent prognostic factor in patients with colorectal cancer, but further comprehensive studies are warranted to determine its optimal values for specific states of the disease.

\section{CONFLICT OF INTEREST}

No potential conflict of interest relevant to this article was reported.

\section{REFERENCES}

1. Berger AC, Sigurdson ER, LeVoyer T, Hanlon A, Mayer RJ, Macdonald JS, et al. Colon cancer survival is associated with decreasing ratio of metastatic to examined lymph nodes. J Clin Oncol 2005;23:8706-12.

2. Johnson PM, Porter GA, Ricciardi R, Baxter NN. Increasing negative lymph node count is independently associated with improved long-term survival in stage IIIB and IIIC colon cancer. J Clin Oncol 2006;24:3570-5.

3. Peng J, Xu Y, Guan Z, Zhu J, Wang M, Cai G, et al. Prognostic sig- 
nificance of the metastatic lymph node ratio in node-positive rectal cancer. Ann Surg Oncol 2008;15:3118-23.

4. Kim YS, Kim JH, Yoon SM, Choi EK, Ahn SD, Lee SW, et al. lymph node ratio as a prognostic factor in patients with stage III rectal cancer treated with total mesorectal excision followed by chemoradiotherapy. Int J Radiat Oncol Biol Phys 2009;74:796-802.

5. Shin JY, Hong KH. Prognostic significance of lymph node ratio in stage III rectal cancer. J Korean Soc Coloproctol 2011;27:252-9.

6. Allaix ME, Arezzo A, Cassoni P, Mistrangelo M, Giraudo G, Morino M. Metastatic lymph node ratio as a prognostic factor after laparoscopic total mesorectal excision for extraperitoneal rectal cancer. Surg Endosc 2013;27:1957-67.

7. Kang J, Hur H, Min BS, Lee KY, Kim NK. Prognostic impact of the lymph node ratio in rectal cancer patients who underwent preoperative chemoradiation. J Surg Oncol 2011;104:53-8.

8. Klos CL, Bordeianou LG, Sylla P, Chang Y, Berger DL. The prognostic value of lymph node ratio after neoadjuvant chemoradiation and rectal cancer surgery. Dis Colon Rectum 2011;54:171-5.
9. Lee SD, Kim TH, Kim DY, Baek JY, Kim SY, Chang HJ, et al. Lymph node ratio is an independent prognostic factor in patients with rectal cancer treated with preoperative chemoradiotherapy and curative resection. Eur J Surg Oncol 2012;38:478-83.

10. Attaallah W, Gunal O, Manukyan M, Ozden g, Yegen C. Prognostic impact of the metastatic lymph node ratio on survival in rectal cancer. Ann Coloproctol 2013;29:100-5.

11. Rosenberg R, Friederichs J, Schuster T, Gertler R, Maak M, Becker $\mathrm{K}$, et al. Prognosis of patients with colorectal cancer is associated with lymph node ratio: a single-center analysis of 3,026 patients over a 25-year time period. Ann Surg 2008;248:968-78.

12. Ceelen W, Van Nieuwenhove Y, Pattyn P. Prognostic value of the lymph node ratio in stage III colorectal cancer: a systematic review. Ann Surg Oncol 2010;17:2847-55.

13. Wong KP, Poon JT, Fan JK, Law WL. Prognostic value of lymph node ratio in stage III colorectal cancer. Colorectal Dis 2011;13: 1116-22. 